\title{
Common fixed points for single-valued and multi-valued maps satisfying a generalized contraction in G-metric spaces
}

\author{
Nedal Tahat ${ }^{1 *}$, Hassen Aydi ${ }^{2}$, Erdal Karapinar ${ }^{3}$ and Wasfi Shatanawi ${ }^{1}$
}

\footnotetext{
* Correspondence: nedal@hu.edu.jo ${ }^{1}$ Department of Mathematics,

Hashemite University, Zarqa 13115, Jordan

Full list of author information is available at the end of the article
}

\begin{abstract}
In this article, we establish some common fixed point theorems for a hybrid pair $\{g$, T\} of single valued and multi-valued maps satisfying a generalized contractive condition defined on G-metric spaces. Our results unify, generalize and complement various known comparable results from the current literature. 2000 MSC: 54H25; 47H10; 54E50.
\end{abstract}

Keywords: multi-valued mappings, common fixed point, weakly compatible mappings, generalized contraction

\section{Introduction and preliminaries}

Nadler [1] initiated the study of fixed points for multi-valued contraction mappings and generalized the well known Banach fixed point theorem. Then after, many authors studied many fixed point results for multi-valued contraction mappings see [2-13].

Mustafa and Sims [14] introduced the G-metric spaces as a generalization of the notion of metric spaces. Mustafa et al. [15-19] obtained some fixed point theorems for mappings satisfying different contractive conditions. Abbas and Rhoades [20] initiated the study of common fixed point in G-metric spaces. While Saadati et al. [21] studied some fixed point theorems in generalized partially ordered G-metric spaces. Gajić and Crvenković [22,23] proved some fixed point results for mappings with contractive iterate at a point in G-metric spaces. For other studies in G-metric spaces, we refer the reader to [24-38]. Consistent with Mustafa and Sims [14], the following definitions and results will be needed in the sequel.

Definition 1.1. (See [14]). Let $X$ be a non-empty set, $G: X \times X \times X \rightarrow \mathbb{R}^{+}$be a function satisfying the following properties

(G1) $G(x, y, z)=0$ if $x=y=z$,

(G2) $0<G(x, x, y)$ for all $x, y \in X$ with $x \neq y$,

(G3) $G(x, x, y) \leq G(x, y, z)$ for all $x, y, z \in X$ with $y \neq z$,

(G4) $G(x, y, z)=G(x, z, y)=G(y, z, x)=\ldots$ (symmetry in all three variables),

(G5) $G(x, y, z) \leq G(x, a, a)+G(a, y, z)$ for all $x, y, z$, a $X$ (rectangle inequality).

Then the function $G$ is called a generalized metric, or, more specially, a G-metric on $X$, and the pair $(X, G)$ is called a G-metric space. 
Definition 1.2. (See [14]). Let $(X, G)$ be a G-metric space, and let $\left(x_{n}\right)$ be a sequence of points of $X$, therefore, we say that $\left(x_{n}\right)$ is G-convergent to $x \in X$ if $\lim _{n, m \rightarrow+\infty} G\left(x, x_{n}, x_{m}\right)=0$, that is, for any $\varepsilon>0$, there exists $N \in \mathbb{N}$ such that $G\left(x, x_{n}, x_{m}\right)$ $<\varepsilon$, for all $n, m \geq N$. We call $x$ the limit of the sequence and write $x_{n} \rightarrow x$ or $\lim _{n \rightarrow+\infty} x_{n}=x$.

Proposition 1.1. (See [14]). Let $(X, G)$ be a G-metric space. The following statements are equivalent:

(1) $\left(x_{n}\right)$ is G-convergent to $x$,

(2) $G\left(x_{w}, x_{w}, x\right) \rightarrow 0$ as $n \rightarrow+\infty$,

(3) $G\left(x_{n}, x, x\right) \rightarrow 0$ as $n \rightarrow+\infty$,

(4) $G\left(x_{n}, x_{m}, x\right) \rightarrow 0$ as $n, m \rightarrow+\infty$.

Definition 1.3. (See [14]). Let $(X, G)$ be a G-metric space. A sequence $\left(x_{n}\right)$ is called a $G$-Cauchy sequence if for any $\varepsilon>0$, there is $N \in \mathbb{N}$ such that $G\left(x_{w}, x_{m}, x_{l}\right)<\varepsilon$ for all $m$, $n, l \geq N$, that is, $G\left(x_{w}, x_{m}, x_{l}\right) \rightarrow 0$ as $n, m, l \rightarrow+\infty$.

Proposition 1.2. (See [14]). Let $(X, G)$ be a G-metric space. Then the following statements are equivalent:

(1) the sequence $\left(x_{n}\right)$ is G-Cauchy,

(2) for any $\varepsilon>0$, there exists $N \in \mathbb{N}$ such that $G\left(x_{w}, x_{m}, x_{m}\right)<\varepsilon$, for all $m, n \geq N$.

Definition 1.4. (See [14]). A G-metric space $(X, G)$ is called G-complete if every $G$ Cauchy sequence is G-convergent in $(X, G)$.

Every G-metric on $X$ defines a metric $d_{G}$ on $X$ given by

$$
d_{G}(x, y)=G(x, y, y)+G(y, x, x), \text { for all } x, y \in X .
$$

Recently, Kaewcharoen and Kaewkhao [34] introduced the following concepts. Let $X$ be a $G$-metric space. We shall denote $C B(X)$ the family of all nonempty closed bounded subsets of $X$. Let $H(. . .$.$) be the Hausdorff G$-distance on $C B(X)$, i.e.,

$$
H_{G}(A, B, C)=\max \left\{\sup _{x \in A} G(x, B, C), \sup _{x \in B} G(x, C, A), \sup _{x \in C} G(x, A, B)\right\},
$$

where

$$
\begin{gathered}
G(x, B, C)=d_{G}(x, B)+d_{G}(B, C)+d_{G}(x, C), \\
d_{G}(x, B)=\inf \left\{d_{G}(x, y), y \in B\right\}, \\
d_{G}(A, B)=\inf \left\{d_{G}(a, b), a \in A, b \in B\right\} .
\end{gathered}
$$

Recall that $G(x, y, C)=\inf \{G(x, y, z), z \in C\}$. A mapping $T: X \rightarrow 2^{X}$ is called a multi-valued mapping. A point $x \in X$ is called a fixed point of $T$ if $x \in T x$.

Definition 1.5. Let $X$ be a given non empty set. Assume that $g: X \rightarrow X$ and $T: X \rightarrow$ $2^{X}$.

If $w=g x \in T x$ for some $x \in X$, then $x$ is called a coincidence point of $g$ and $T$ and $w$ is a point of coincidence of $g$ and $T$.

Mappings $g$ and $T$ are called weakly compatible if $g x \in T x$ for some $x \in X$ implies $g T$ $(x) \subseteq \operatorname{Tg}(x)$.

Proposition 1.3. (see [34]). Let $X$ be a given non empty set. Assume that $g: X \rightarrow X$ and $T: X \rightarrow 2^{X}$ are weakly compatible mappings. If $g$ and $T$ have a unique point of coincidence $w=g x \in T x$, then $w$ is the unique common fixed point of $g$ and $T$. 
In this article, we establish some common fixed point theorems for a hybrid pair $\{g$, $T\}$ of single valued and multi-valued maps satisfying a generalized contractive condition defined on G-metric spaces. Also, an example is presented.

\section{Main results}

We start this section with the following lemma, which is the variant of the one given in Nadler [1] or Assad and Kirk [4]. Its proof is a simple consequence of the definition of the Hausdorff $G$-distance $H_{G}(A, B, B)$.

Lemma 2.1. If $A, B \in C B(X)$ and $a \in A$, then for each $\varepsilon>0$, there exists $b \in B$ such that $G(a, b, b) \leq H_{G}(A, B, B)+\varepsilon$.

The main result of the article is the following.

Theorem 2.1. Let $(X, G)$ be a G-metric space. Set $g: X \rightarrow X$ and $T: X \rightarrow C B(X)$. Assume that there exists a function $\alpha:[0,+\infty) \rightarrow[0,1)$ satisfying $\limsup _{r \rightarrow t^{+}} \alpha(r)<1_{\text {for }}$ every $t \geq 0$ such that

$$
H_{G}(T x, T y, T z) \leq \alpha(G(g x, g y, g z)) G(g x, g y, g z),
$$

for all $x, y, z \in X$. If for any $x \in X, T x \subseteq g(X)$ and $g(X)$ is a G-complete subspace of $X$, then $g$ and $T$ have a point of coincidence in $X$. Furthermore, if we assume that gp $\in$ $T p$ and $g q \in T q$ implies $G(g q, g p, g p) \leq H_{G}(T q, T p, T p)$, then

(i) $g$ and $T$ have a unique point of coincidence.

(ii) If in addition $g$ and $T$ are weakly compatible, then $g$ and $T$ have a unique common fixed point.

Proof. Let $x_{0}$ be arbitrary in $X$. Since $T x_{0} \subseteq g(X)$, choose $x_{1} \in X$ such that $g x_{1} \in T x_{0}$. If $g x_{1}=g x_{0}$, we finished. Assume that $g x_{0} \neq g x_{1}$, so $G\left(g x_{0}, g x_{1}, g x_{1}\right)>0$. We can choose a positive integer $n_{1}$ such that

$$
\alpha^{n_{1}}\left(G\left(g x_{0}, g x_{1}, g x_{1}\right)\right) \leq\left[1-\alpha\left(G\left(g x_{0}, g x_{1}, g x_{1}\right)\right)\right] G\left(g x_{0}, g x_{1}, g x_{1}\right) .
$$

By Lemma 2.1 and the fact that $T x_{1} \subseteq g(X)$, there exists $g x_{2} \in T x_{1}$ such that

$$
G\left(g x_{1}, g x_{2}, g x_{2}\right) \leq H_{G}\left(T x_{0}, T x_{1}, T x_{1}\right)+\alpha^{n_{1}}\left(G\left(g x_{0}, g x_{1}, g x_{1}\right)\right) .
$$

Using the two above inequalities and (2), it follows that

$$
\begin{aligned}
G\left(g x_{1}, g x_{2}, g x_{2}\right) & \leq H_{G}\left(T x_{0}, T x_{1}, T x_{1}\right)+\alpha^{n_{1}}\left(G\left(g x_{0}, g x_{1}, g x_{1}\right)\right) \\
& \leq \alpha\left(G\left(g x_{0}, g x_{1}, g x_{1}\right)\right) G\left(g x_{0}, g x_{1}, g x_{1}\right)+\left[1-\alpha\left(G\left(g x_{0}, g x_{1}, g x_{1}\right)\right)\right] G\left(g x_{0}, g x_{1}, g x_{1}\right) \\
& =G\left(g x_{0}, g x_{1}, g x_{1}\right) .
\end{aligned}
$$

If $g x_{1}=g x_{2}$, we finished. Assume that $g x_{1} \neq g x_{2}$. Now we choose a positive integer $n_{2}>n_{1}$ such that

$$
\alpha^{n_{2}}\left(G\left(g x_{1}, g x_{2}, g x_{2}\right)\right) \leq\left[1-\alpha\left(G\left(g x_{1}, g x_{2}, g x_{2}\right)\right)\right] G\left(g x_{2}, g x_{2}, g x_{2}\right) .
$$

Since $T x_{2} \in C B(X)$ and the fact that $T x_{2} \subseteq g(X)$, we may select $g x_{3} \in T x_{2}$ such that from Lemma 2.1

$$
G\left(g x_{2}, g x_{3}, g x_{3}\right) \leq H_{G}\left(T x_{1}, T x_{2}, T x_{2}\right)+\alpha^{n_{2}}\left(G\left(g x_{1}, g x_{2}, g x_{2}\right)\right),
$$


and then, similarly to the previous case, we have

$$
\begin{aligned}
G\left(g x_{2}, g x_{3}, g x_{3}\right) & \leq H_{G}\left(T x_{1}, T x_{2}, T x_{2}\right)+\alpha^{n_{2}}\left(G\left(g x_{1}, g x_{2}, g x_{2}\right)\right) \\
& \leq \alpha\left(G\left(g x_{1}, g x_{2}, g x_{2}\right)\right) G\left(g x_{1}, g x_{2}, g x_{2}\right)+\left[1-\alpha\left(G\left(g x_{1}, g x_{2}, g x_{2}\right)\right)\right] G\left(g x_{1}, g x_{2}, g x_{2}\right) \\
& =G\left(g x_{1}, g x_{2}, g x_{2}\right) .
\end{aligned}
$$

By repeating this process, for each $k \in \mathbb{N}^{*}$, we may choose a positive integer $n_{k}$ such that

$$
\alpha^{n_{k}}\left(G\left(g x_{k-1}, g x_{k}, g x_{k}\right)\right) \leq\left[1-\alpha\left(G\left(g x_{k-1}, g x_{k}, g x_{k}\right)\right)\right] G\left(g x_{k-1}, g x_{k}, g x_{k}\right) .
$$

Again, we may select $g x_{k+1} \in T x_{k}$ such that

$$
G\left(g x_{k}, g x_{k+1}, g x_{k+1}\right) \leq H_{G}\left(T x_{k-1}, T x_{k}, T x_{k}\right)+\alpha^{n_{k}}\left(G\left(g x_{k-1}, g x_{k}, g x_{k}\right)\right) .
$$

The last two inequalities together imply that

$$
G\left(g x_{k}, g x_{k+1}, g x_{k+1}\right) \leq G\left(g x_{k-1}, g x_{k}, g x_{k}\right)
$$

which shows that the sequence of nonnegative numbers $\left\{d_{k}\right\}$, given by $d_{k}=G\left(g x_{k-1}\right.$, $\left.g x_{k} g x_{k}\right), k=1,2, \ldots$, is non-increasing. This means that there exists $d \geq 0$ such that

$$
\lim _{k \rightarrow+\infty} d_{k}=d \text {. }
$$

Let now prove that the $\left\{g x_{k}\right\}$ is a G-Cauchy sequence.

Using the fact that, by hypothesis for $t=d, \limsup _{r \rightarrow d^{+}} \alpha(t)<1$, it results that there exists a rank $k_{0}$ such that for $k \geq k_{0}$, we have $\alpha\left(d_{k}\right)<h$, where

$$
\limsup _{t \rightarrow d^{+}} \alpha(t)<h<1 \text {. }
$$

Now, by (3) we deduce that the sequence $\left\{d_{k}\right\}$ satisfies the following recurrence inequality

$$
d_{k+1} \leq H_{G}\left(T x_{k-1}, T x_{k}, T x_{k}\right)+\alpha^{n_{k}}\left(d_{k}\right) \leq \alpha\left(d_{k}\right) d_{k}+\alpha^{n_{k}}\left(d_{k}\right), \quad k \geq 1 .
$$

By induction, from (4), we get

$$
d_{k+1} \leq \prod_{i=1}^{k} \alpha\left(d_{i}\right) d_{1}+\sum_{m=1}^{k-1} \prod_{i=m+1}^{k} \alpha\left(d_{i}\right) \alpha^{n_{m}}\left(d_{m}\right)+\alpha^{n_{k}}\left(d_{k}\right), \quad k \geq 1,
$$

which, by using the fact that $\alpha<1$, can be simplified to

$$
d_{k+1} \leq \prod_{i=1}^{k} \alpha\left(d_{i}\right) d_{1}+\sum_{m=1}^{k-1} \prod_{i=\max \left\{k_{0}, m+1\right\}}^{k} \alpha\left(d_{i}\right) \alpha^{n_{m}}\left(d_{m}\right)+\alpha^{n_{k}}\left(d_{k}\right), \quad k \geq 1,
$$

Referring to the proof of Theorem 2.1 in [11] or Lemma 3.2 in [12], we may obtain

$$
\prod_{i=1}^{k} \alpha\left(d_{i}\right) d_{1}+\sum_{m=1}^{k-1} \prod_{i=\max \left\{k_{0}, m+1\right\}}^{k} \alpha\left(d_{i}\right) \alpha^{n_{m}}\left(d_{m}\right)+\alpha^{n_{k}}\left(d_{k}\right) \leq c h^{k},
$$

where $c$ is a positive constant. We deduce that

$$
d_{k+1}=G\left(g x_{k}, g x_{k+1}, g x_{k+1}\right) \leq c h^{k} .
$$


Now for $k \geq k_{0}$ and $m$ is a positive arbitrary integer, we have using the property (G4)

$$
\begin{aligned}
G\left(g x_{k}, g x_{k+m}, g x_{k+m}\right) \leq & G\left(g x_{k}, g x_{k+1}, g x_{k+1}\right)+G\left(g x_{k+1}, g x_{k+2}, g x_{k+2}\right) \\
& +\cdots+G\left(g x_{k+m-2}, g x_{k+m-1}, g x_{k+m-1}\right)+G\left(g x_{k+m-1}, g x_{k+m}, g x_{k+m}\right) \\
\leq & c\left[h^{k}+h^{k+1}+\cdots+h^{k+m-1}\right] \\
\leq & c \frac{h^{k}}{1-h} \rightarrow 0 \text { as } k \rightarrow+\infty,
\end{aligned}
$$

since $0<h<1$. This shows that the sequence $\left\{g x_{n}\right\}$ is G-Cauchy in the complete subspace $g(X)$. Thus, there exists $q \in g(X)$ such that, from Proposition 1.1

$$
\lim _{n \rightarrow+\infty} G\left(g x_{n}, g x_{n}, q\right)=\lim _{n \rightarrow+\infty} G\left(g x_{n}, q, q\right)=0 .
$$

Since $q \in g(X)$, then there exists $p \in X$ such that $q=g p$. From (5), we have

$$
\lim _{n \rightarrow+\infty} G\left(g x_{n}, g x_{n}, g p\right)=\lim _{n \rightarrow+\infty} G\left(g x_{n}, g p, g p\right)=0 .
$$

We claim that $g p \in T p$. Indeed, from (2), we have

$$
\begin{aligned}
G\left(g x_{n+1}, T p, T p\right) & \leq H_{G}\left(T x_{n}, T p, T p\right) \\
& \leq \alpha\left(G\left(g x_{n}, g p, g p\right)\right) G\left(g x_{n}, g p, g p\right) .
\end{aligned}
$$

Letting $n \rightarrow+\infty$ in (7) and using (6), we get

$$
G(g p, T p, T p)=\lim _{n \rightarrow+\infty} G\left(g x_{n+1}, T p, T p\right)=0,
$$

that is, $g p \in T p$. That is $T$ and $g$ have a point of coincidence. Now, assume that if $g p$ $\in T p$ and $g q \in T q$, then $G(g q, g p, g p) \leq H_{G}(T q, T p, T p)$. We will prove the uniqueness of a point of coincidence of $g$ and $T$. Suppose that $g p \in T p$ and $g q \in T q$. By (2) and this assumption, we have

$$
\begin{aligned}
G(g q, g p, g p) & \leq H_{G}(T q, T p, T p) \\
& \leq \alpha(G(g q, g p, g p)) G(g q, g p, g p),
\end{aligned}
$$

and since $\alpha(G(g q, g p, g p))<G(g q, g p, g p)$, so necessarily from (8), we have $G(g q, g p$, $g p)=0$, i.e., $g p=g q$. In view of

$$
H_{G}(T q, T p, T p) \leq \alpha(G(g q, g p, g p)) G(g q, g p, g p)=0,
$$

we get $T q=T p$. Thus, $T$ and $g$ have a unique point of coincidence. Suppose that $g$ and $T$ are weakly compatible. By applying Proposition 1.3, we obtain that $g$ and $T$ have a unique common fixed point.

Corollary 2.1. Let $(X, G)$ be a complete G-metric space. Assume that $T: X \rightarrow C B(X)$ satisfies the following condition

$$
H_{G}(T x, T y, T z) \leq \alpha(G(x, y, z)) G(x, y, z)
$$

for all $x, y, z \in X$, where $\alpha:[0,+\infty) \rightarrow[0,1)$ satisfies $\limsup _{r \rightarrow t^{+}} \alpha(r)<1_{\text {for }}$ every $t \geq 0$. Then $T$ has a fixed point in X. Furthermore, if we assume that $p \in T p$ and $q \in T q$ implies $G(q, p, p) \leq H_{G}(T q, T p$, Tp $)$, then $T$ has a unique fixed point.

Proof. It follows by taking $g$ the identity on $X$ in Theorem 2.1 .

Corollary 2.2. Let $(X, G)$ be a G-metric space. Assume that $g: X \rightarrow X$ and $T: X \rightarrow$ $C B(X)$ satisfy the following condition 


$$
H_{G}(T x, T y, T z) \leq k G(g x, g y, g z),
$$

for all $x, y, z \in X$, where $k \in[0,1)$. If for any $x \in X, T x \subseteq g(X)$ and $g(X)$ is a G-complete subspace of $X$, then $g$ and $T$ have a point of coincidence in $X$. Furthermore, if we assume that $g p \in T p$ and $g q \in T q$ implies $G(g q, g p, g p) \leq H_{G}(T q, T p, T p)$, then

(i) $g$ and $T$ have a unique point of coincidence.

(ii) If in addition $g$ and $T$ are weakly compatible, then $g$ and $T$ have a unique common fixed point.

Proof. It follows by taking $\alpha(t)=k, k \in[0,1)$, in Theorem 2.1.

In the case of single-valued mappings, that is, if $T: X \rightarrow X$, (i.e., $T x=\{T x\}$ for any $x$ $\in X$ ), it is obviously that

$$
H_{G}(T x, T y, T z)=G(T x, T y, T z), \quad \forall x, y, z \in X .
$$

Furthermore, if $g p \in T p$ (i.e., $g p=T p$ ) and $g q \in T q$ (i.e., $g q=T q$ ), then clearly,

$$
G(g q, g p, g p)=G(T q, T p, T p)=H_{G}(T q, T p, T p),
$$

that is, the assumption given in Theorem 2.1 is verified.

Also, the single-valued mappings $T, g: X \rightarrow X$ are said weakly compatible if $T g x=$ $g T x$ whenever $T x=g x$ for some $x \in X$.

Now, we may state the following corollaries from Theorem 2.1 and the precedent corollaries:

Corollary 2.3. Let $(X, G)$ be a complete G-metric space. Assume that $T: X \rightarrow X$ satisfies the following condition

$$
G(T x, T y, T z) \leq \alpha(G(x, y, z)) G(x, y, z)
$$

for all $x, y, z \in X$, where $\alpha:[0,+\infty) \rightarrow[0,1)$ satisfies $\limsup _{r \rightarrow t^{+}} \alpha(r)<1_{\text {for }}$ every $t \geq 0$. Then, $T$ has a unique fixed point.

Corollary 2.4. Let $(X, G)$ be a G-metric space. Assume that $g: X \rightarrow X$ and $T: X \rightarrow$ $X$ satisfy the following condition

$$
G(T x, T y, T z) \leq \alpha(G(g x, g y, g z)) G(g x, g y, g z)
$$

for all $x, y, z \in X$, where $\alpha:[0,+\infty) \rightarrow[0,1)$ satisfies $\limsup _{r \rightarrow t^{+}} \alpha(r)<1_{\text {for every }} t \geq 0$. If $T(X) \subseteq g(X)$ and $g(X)$ is a G-complete subspace of $X$, then

(i) $g$ and $T$ have a unique point of coincidence.

(ii) Furthermore, if $g$ and $T$ are weakly compatible, then $g$ and $T$ have a unique common fixed point.

Now, we introduce an example to support the useability of our results.

Example 2.1. Let $X=[0,1]$. Define $T: X \rightarrow C B(X)$ by $T x=\left[0, \frac{1}{16} x\right]$ and define $g: X$ $\rightarrow X$ by $g x=\sqrt{x}$. Define a G-metric on $X$ by $G(x, y, z)=\max \{|x-y|,|x-z|,|y-z|\}$. Also, define $\alpha:[0,+\infty) \rightarrow[0,1)$ by $\alpha(t)=\frac{1}{2}$ Then:

(1) $T x \subseteq g(X)$ for all $x \in X$.

(2) $g(X)$ is a G-complete subspace of $X$.

(3) $g$ and $T$ are weakly compatible.

(4) $H_{G}(T x, T y, T z) \leq \alpha(G(g x, g y, g z)) G(g x, g y, g z)$ for all $x, y, z \in X$. 
Proof. The proofs of (1), (2), and (3) are clear. By (1), we have

$$
d_{G}(x, y)=G(x, y, y)+G(y, x, x)=2|x-y| \text { for all } x, y \in X .
$$

To prove (4), let $x, y, z \in X$. If $x=y=z=0$, then

$$
H_{G}(T x, T y, T z)=0 \leq \alpha(G(g x, g y, g z)) G(g x, g y, g z) .
$$

Thus, we may assume that $x, y$, and $z$ are not all zero. With out loss of generality, we assume that $x \leq y \leq z$. Then

$$
\begin{aligned}
& H_{G}(T x, T y, T z)=H_{G}\left(\left[0, \frac{1}{16} x\right],\left[0, \frac{1}{16} y\right],\left[0, \frac{1}{16} z\right]\right)
\end{aligned}
$$

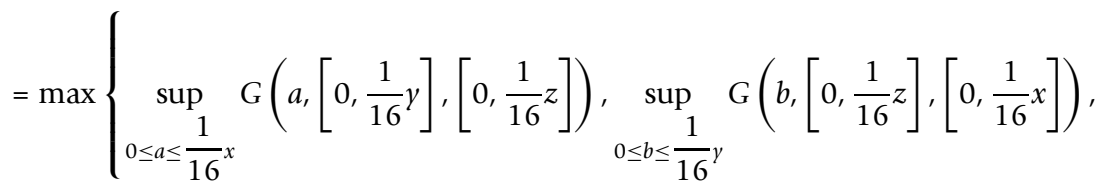

$$
\begin{aligned}
& \left.\sup _{0 \leq c \leq \frac{1}{16} z} G\left(c,\left[0, \frac{1}{16} x\right],\left[0, \frac{1}{16} y\right]\right)\right\} \text {. }
\end{aligned}
$$

Since $x \leq y \leq z$, so $\left[0, \frac{1}{16} x\right] \subseteq\left[0, \frac{1}{16} y\right] \subseteq\left[0, \frac{1}{16} z\right]$ This implies that

$$
d_{G}\left(\left[0, \frac{1}{16} x\right],\left[0, \frac{1}{16} y\right]\right)=d_{G}\left(\left[0, \frac{1}{16} y\right],\left[0, \frac{1}{16} z\right]\right)=d_{G}\left(\left[0, \frac{1}{16} x\right],\left[0, \frac{1}{16} z\right]\right)=0 .
$$

For each $0 \leq a \leq \frac{1}{16} x$, we have

$$
G\left(a_{1}\left[0, \frac{1}{16} y\right],\left[0, \frac{1}{16} z\right]\right)=d_{G}\left(a,\left[0, \frac{1}{16} y\right]\right)+d_{G}\left(\left[0, \frac{1}{16} y\right],\left[0, \frac{1}{16} z\right]\right)+d_{G}\left(a,\left[0, \frac{1}{16} z\right]\right)=0 .
$$

Also, for each $0 \leq b \leq \frac{1}{16} y$, we have

$$
\begin{aligned}
G\left(b,\left[0, \frac{1}{16} z\right],\left[0, \frac{1}{16} x\right]\right) & =d_{G}\left(b,\left[0, \frac{1}{16} z\right]\right)+d_{G}\left(\left[0, \frac{1}{16} z\right],\left[0, \frac{1}{16} x\right]\right)+d_{G}\left(b,\left[0, \frac{1}{16} x\right]\right) \\
& =\left\{\begin{array}{l}
0 \text { if } b \leq \frac{x}{16} \\
2 b-\frac{x}{8} \text { if } b \geq \frac{x}{16} .
\end{array}\right.
\end{aligned}
$$

This yields that

$$
\sup _{0 \leq b \leq \frac{1}{16} y} G\left(b,\left[0, \frac{1}{16} z\right],\left[0, \frac{1}{16} x\right]\right)=\frac{y}{8}-\frac{x}{8} .
$$


Moreover, for each $0 \leq c \leq \frac{1}{16} z$, we have

$$
\begin{aligned}
G\left(c,\left[0, \frac{1}{16} x\right],\left[0, \frac{1}{16} y\right]\right) & =d_{G}\left(c,\left[0, \frac{1}{16} x\right]\right)+d_{G}\left(\left[0, \frac{1}{16} x\right],\left[0, \frac{1}{16} y\right]\right)+d_{G}\left(c,\left[0, \frac{1}{16} y\right]\right) \\
= & \left\{\begin{array}{l}
0 \text { if } c \leq \frac{x}{16} x \\
2 c-\frac{x}{8} \text { if } \frac{x}{16} \leq c \leq \frac{y}{16} \\
4 c-\frac{x}{8}-\frac{y}{8} \text { if } c \geq \frac{y}{16} .
\end{array}\right.
\end{aligned}
$$

This yields that

$$
\sup _{0 \leq c \leq \frac{1}{16} z} G\left(c,\left[\frac{1}{16} c\right],\left[0, \frac{1}{16} y\right]\right)=\frac{z}{4}-\frac{x}{8}-\frac{y}{8} .
$$

We deduce that

$$
\begin{aligned}
H_{G}(T x, T y, T z) & =\frac{z}{4}-\frac{x}{8}-\frac{y}{8} \\
& \leq \frac{1}{4}(z-x) \\
& =\frac{1}{2}\left(\frac{1}{2}(z-x)\right) \\
& \leq \frac{1}{2}\left(\frac{z-x}{\sqrt{x}+\sqrt{z}}\right) \\
& =\frac{1}{2}(\sqrt{z}-\sqrt{x})
\end{aligned}
$$

On the other hand, it is obvious that all other hypotheses of Theorem 2.1 are satisfied and so $g$ and $T$ have a unique common fixed point, which is $u=0$.

Remark 1. Theorem 2.1 improves Kaewcharoen and Kaewkhao [[34], Theorem 3.3] (in case $b=c=d=0$ ).

- Corollary 2.3 generalizes Mustafa [[15], Theorem 5.1.7] and Shatanawi [[35], Corollary 3.4].

\section{Acknowledgements}

The authors thank the editor and the referees for their useful comments and suggestions.

\section{Author details}

${ }^{1}$ Department of Mathematics, Hashemite University, Zarqa 13115, Jordan ²Université de Sousse, Institut Supérieur d'Informatique et des Technologies de Communication De Hammam Sousse, Route GP1-4011, Hammam Sousse, Tunisie ${ }^{3}$ Department of Mathematics, Atilim University 06836, İncek, Ankara, Turkey

\section{Authors' contributions}

The authors have contributed in obtaining the new results presented in this article. All authors read and approved the final manuscript.

\section{Competing interests}

The authors declare that they have no competing interests.

Received: 18 October 2011 Accepted: 26 March 2012 Published: 26 March 2012

\section{References}

1. Nadler, SB: Multi-valued contraction mappings. Pacific J Math. 30, 475-478 (1969)

2. Gorniewicz, L: Topological fixed point theory of multivalued mappings. Kluwer Academic Publishers, Dordrecht (1999) 
3. Klim, D, Wardowski, D: Fixed point theorems for set-valued contractions in complete metric spaces. J Math Anal Appl. 334, 132-139 (2007). doi:10.1016/j.jmaa.2006.12.012

4. Assad, NA, Kirk, WA: Fixed point theorems for setvalued mappings of contractive type. Pacific J Math. 43, 553-562 (1972)

5. Hong, SH: Fixed points of multivalued operators in ordered metric spaces with applications. Nonlinear Anal. 72, 3929-3942 (2010). doi:10.1016/j.na.2010.01.013

6. Hong, SH: Fixed points for mixed monotone multivalued operators in Banach Spaces with applications. J Math Anal Appl. 337, 333-342 (2008). doi:10.1016/j.jmaa.2007.03.091

7. Hong, SH, Guan, D, Wang, L: Hybrid fixed points of multivalued operators in metric spaces with applications. Nonlinear Anal. 70, 4106-4117 (2009). doi:10.1016/.na.2008.08.020

8. Hong, SH: Fixed points of discontinuous multivalued increasing operators in Banach spaces with applications. J Math Anal Appl. 282, 151-162 (2003). doi:10.1016/S0022-247X(03)00111-2

9. Shatanawi, W: Some fixed point results for a generalized $\psi$-weak contraction mappings in orbitally metric spaces. Chaos Solitons Fract. 45, 520-526 (2012). doi:10.1016/j.chaos.2012.01.015

10. Mizoguchi, N, Takahashi, W: Fixed point theorems for multi-valued mappings on complete metric spaces. J Math Anal Appl. 141, 177-188 (1989). doi:10.1016/0022-247X(89)90214-X

11. Berinde, $M$, Berinde, V: On a general class of multi-valued weakly Picard mappings. J Math Anal Appl. 326, 772-782 (2007). doi:10.1016/j.jmaa.2006.03.016

12. Kamran, T: Multivalued f-weakly Picard mappings. Nonlinear Anal. 67, 2289-2296 (2007). doi:10.1016/..na.2006.09.010

13. Al-Thagafi, MA, Shahzad, N: Coincidence points, generalized I-nonexpansive multimaps and applications. Nonlinear Anal. 67, 2180-2188 (2007). doi:10.1016/j.na.2006.08.042

14. Mustafa, Z, Sims, B: A new approach to generalized metric spaces. J Nonlinear Convex Anal. 7, 289-297 (2006)

15. Mustafa, Z: A new structure for generalized metric spaces with applications to fixed point theory. University of Newcastle, Newcastle, UK (2005). Ph.D. thesis

16. Mustafa, Z, Obiedat, H, Awawdeh, F: Some fixed point theorem for mapping on complete G-metric spaces. Fixed Point Theory Appl 2008, 12 (2008). ID 189870

17. Mustafa, Z, Sims, B: Fixed point theorems for contractive mappings in complete G-metric spaces. Fixed Point Theory Appl 2009, 10 (2009). ID 917175

18. Mustafa, Z, Khandaqji, M, Shatanawi, W: Fixed point results on complete G-metric spaces. Studia Scientiarum Mathematicarum Hungarica. 48, 304-319 (2011). doi:10.1556/SScMath.48.2011.3.1170

19. Mustafa, Z, Shatanawi, W, Bataineh, M: Existence of fixed point results in G-metric spaces. Int J Math Math Sci 2009, 10 (2009). ID 283028

20. Abbas, M, Rhoades, BE: Common fixed point results for non-commuting mappings without continuity in generalized metric spaces. Appl Math Comput. 215, 262-269 (2009). doi:10.1016/j.amc.2009.04.085

21. Saadati, R, Vaezpour, SM, Vetro, P, Rhoades, BE: Fixed point theorems in generalized partially ordered G-metric spaces. Math Comput Model. 52, 797-801 (2010). doi:10.1016/j.mcm.2010.05.009

22. Gajić, L, Crvenković, ZL: On mappings with contractive iterate at a point in generalized metric spaces. Fixed Point Theory Appl 2010 (2010). (ID 458086), 16 (2010). doi:10.1155/2010/458086

23. Gajić, L, Crvenković, ZL: A fixed point result for mappings with contractive iterate at a point in G-metric spaces. Filomat 25, 53-58 (2011). doi:10.2298/FIL1102053G

24. Abbas, M, Khan, SH, Nazir, T: Common fixed points of R-weakly commuting maps in generalized metric space. Fixed Point Theory Appl. 2011, 41 (2011). doi:10.1186/1687-1812-2011-41

25. Abbas, M, Khan, AK, Nazir, T: Coupled common fixed point results in two generalized metric spaces. Appl Math Comput (2011). doi:10.1016/j.amc.2011.01.006

26. Abbas, M, Nazir, T, Vetro, P: Common fixed point results for three maps in G- metric spaces. Filomat. 25, 1-17 (2011)

27. Aydi, H, Damjanović, B, Samet, B, Shatanawi, W: Coupled fixed point theorems for nonlinear contractions in partially ordered G-metric spaces. Math Comput Model. 54, 2443-2450 (2011). doi:10.1016/j.mcm.2011.05.059

28. Aydi, H, Shatanawi, W, Vetro, C: On generalized weakly G-contraction mapping in G-metric spaces. Comput Math Appl. 62, 4222-4229 (2011). doi:10.1016/j.camwa.2011.10.007

29. Aydi, $H$, Shatanawi, W, Postolache, M: Coupled fixed point results for $(\psi, \varphi)$-weakly contractive mappings in ordered Gmetric spaces. Comput Math Appl. 63, 298-309 (2012). doi:10.1016/j.camwa.2011.11.022

30. Cho, YJ, Rhoades, BE, Saadati, R, Samet, B, Shatanawi, W: Nonlinear coupled fixed point theorems in ordered generalized metric spaces with integral type. Fixed Point Theory and Appl. 2012, 8 (2012). doi:10.1186/1687-1812-2012-8

31. Choudhury, BS, Maity, P: Coupled fixed point results in generalized metric spaces. Math Comput Model. 54, 73-79 (2011). doi:10.1016/.mcm.2011.01.036

32. Chugh, R, Kadian, T, Rani, A, Rhoades, BE: Property P in G-metric spaces. Fixed Point Theory Appl 2010, 12 (2010). (ID 401684)

33. Gholizadeh, L, Saadati, R, Shatanawi, W, Vaezpour, SM: Contractive Mapping in Generalized, Ordered Metric Spaces with Application in Integral Equations. Math Probl Eng 2011, 14 (2011). (ID 380784)

34. Kaewcharoen, A, Kaewkhao, A: Common fixed points for single-valued and multi-valued mappings in G-metric spaces. Int J Math Anal. 5, 1775-1790 (2011)

35. Shatanawi, W: Fixed point theory for contractive mappings satisfying $\Phi$-maps in G-metric spaces. Fixed Point Theory Appl 2010, 9 (2010). (ID 181650)

36. Shatanawi, W: Some fixed point theorems in ordered G-metric spaces and applications. Abst Appl Anal 2011, 11 (2011). (ID 126205)

37. Shatanawi, W: Coupled fixed point theorems in generalized metric spaces. Hacettepe J Math Stat. 40, 441-447 (2011)

38. Shatanawi, W, Abbas, M, Nazir, T: Common coupled coincidence and coupled fixed point results in two generalized metric spaces. Fixed point Theory Appl. 2011, 80 (2011). doi:10.1186/1687-1812-2011-80

doi:10.1186/1687-1812-2012-48

Cite this article as: Tahat et al:: Common fixed points for single-valued and multi-valued maps satisfying a

generalized contraction in G-metric spaces. Fixed Point Theory and Applications 2012 2012:48. 